\title{
Modified autotransplant with three-dimensional printing for treatment of primary cardiac sarcoma
}

\author{
Willian R. Menegazzo, MD, ${ }^{\mathrm{a}}$ Juglans Alvarez, MD, MSc, ${ }^{\mathrm{b}}$ Robert J. Cusimano, MD, MSc, \\ Guilherme Geib, MD, MSc, ${ }^{\mathrm{c}}$ Felipe S. Torres, MD, PhD, ${ }^{\mathrm{d}}$ Nadine Clausell, MD, PhD, ${ }^{\mathrm{a}}$ and \\ Livia A. Goldraich, MD, MSc, ${ }^{\text {a }}$ Porto Alegre, Brazil, and Toronto, Ontario, Canada
}

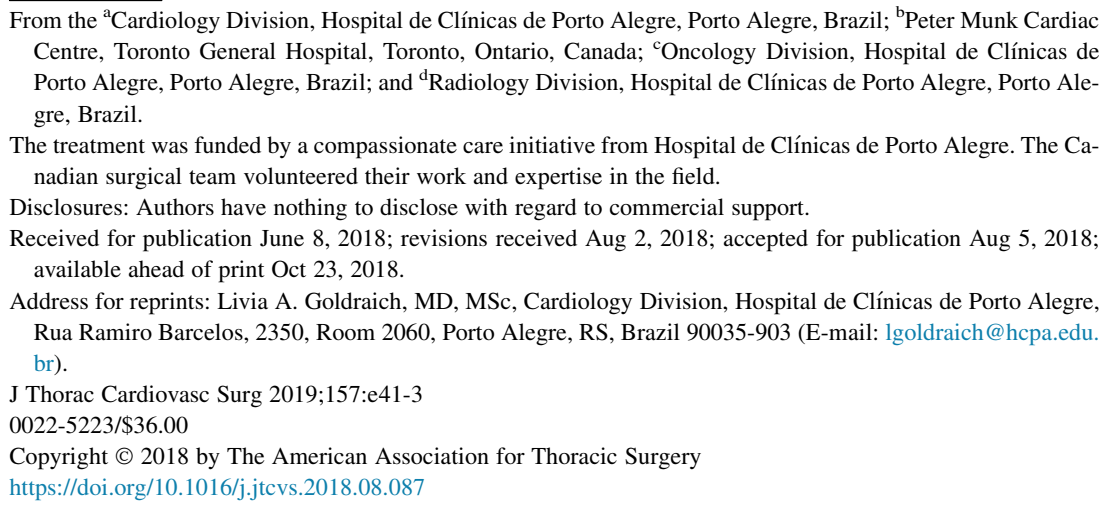

Video clip is available online.

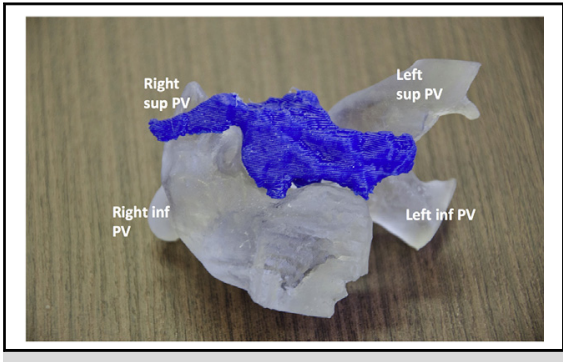

Three-dimensional print modeling for cardiac sarcoma surgical resection.

Central Message

After reconstruction of cardiac structures with a 3-dimensional printing model, a modified autotransplant with resection of the tumor was performed, followed by reconstruction and reimplantation.

See Editorial Commentary page e45.
The treatment of primary cardiac tumors is challenging and requires the expertise of experienced surgeons and a multidisciplinary team. The therapeutic approach should focus on complete tumor resection, which is the major factor associated with prolonged survival. ${ }^{1,2}$ Complex surgical techniques and innovative technologies have been increasingly used by experienced centers to perform complete cardiac tumor resections. ${ }^{3,4}$ We describe here a case of complete resection of primary left-heart sarcoma with a modified autotransplant technique and left atrial reconstruction with 3-dimensional (3D) printing.

\section{CASE DESCRIPTION}

A 20-year-old man presented with $12-\mathrm{kg}$ weight loss and progressive exertional dyspnea during the course of 7 months. Echocardiography and computed tomography revealed a myxoma-appearing bilobed large mass in the left atrium, which was surgically resected. Histopathologic examination showed a high-grade monophasic synovial sarcoma; symptoms and tumor recurred within 30 days, suggesting an incomplete initial resection. Repeat computed tomographic scans showed a $7.4 \times 7.1-\mathrm{cm}$ tumor filling almost the entire left atrium, in close contact with its basal segment and in no surgical plane with its posterior wall (Figure 1, $A-C$ ). There was no evidence of metastatic disease. After careful evaluation, the cardiac oncology surgeon considered the tumor to be resectable by the autotransplant technique. Neoadjuvant chemotherapy was performed with doxorubicin and ifosfamide. After 4 cycles of chemotherapy, the mass size reduced to $2.5 \mathrm{~cm}$, and repeat imaging staging showed no metastasis or esophageal invasion, which was ruled out by endoscopic ultrasonography.

After examination of a $3 \mathrm{D}$ printed model of the mass within adjacent cardiac structures (Figure 1,D), the patient underwent complete tumor resection with modified autotransplantation. The patient was placed on cardiopulmonary bypass after arterial cannulation of the proximal aortic arch and bicaval venous cannulation. Cardioplegic solution (Custodio HTK Solution; Dr Franz Kohler Chemie GMBH, Bensheim, Germany) was delivered in an antegrade fashion through the aortic root. The tumor was easily identified, exactly at the topographical location indicated by imaging. The 3D model precisely matched intraoperative findings. The autotransplantation surgical approach described by Ramlawi and coworkers ${ }^{3}$ was adapted to shorten the ischemic time, and the sequence of surgical maneuvers was planned according to the 3D model (Video 1).

The modified autotransplantation approach was performed through transection of superior vena cava, aorta, 


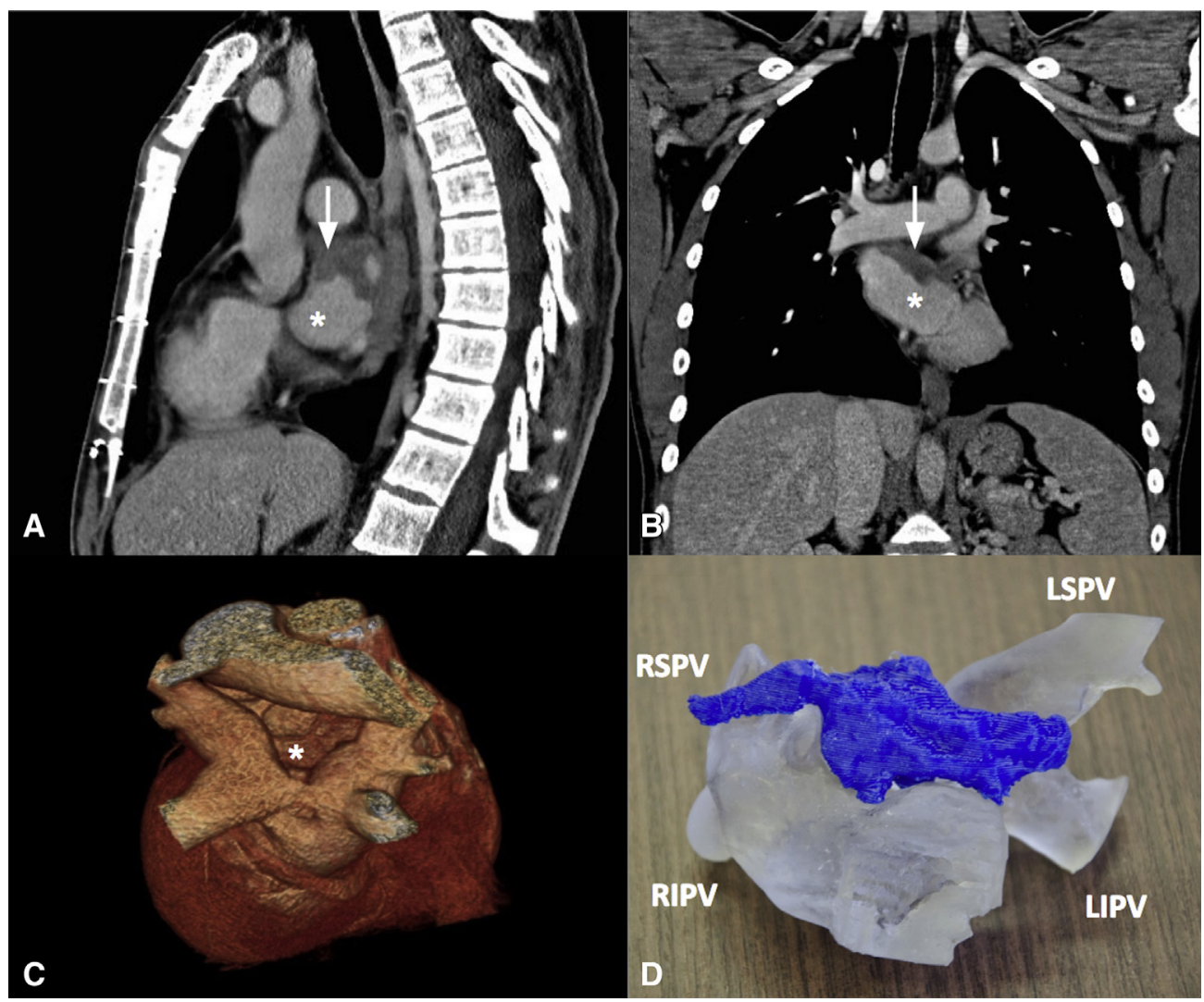

FIGURE 1. A and B, Sagittal (A) and coronal (B) reformatted computed tomographic images at the level of the left atrium (asterisk), demonstrating the location of the tumor at the superior and anterior aspect of the left atrium (arrow). C, Posterior view of a 3-dimensional volume-rendered computed tomographic image of the heart demonstrating the location of the tumor in the left atrium (asterisk). D, Three-dimensional printing of the left atrium with the tumor mass (blue mold) located in the left atrium, leaning toward the mitral valve. $L S P V$, Left superior pulmonary vein; $R S P V$, right superior pulmonary vein; $R I P V$, right inferior pulmonary vein; $L I P V$, left inferior pulmonary vein.

and pulmonary artery, thus gaining access to the left atrial roof and subsequently all posterior and inferior aspects of left atrium, with isolation and control of all 4 pulmonary veins. Complete tumor resection was accomplished, and frozen sections of all margins (roof of left atrium, left atrial appendage, 4 pulmonary vein cuffs, and left atrial margin

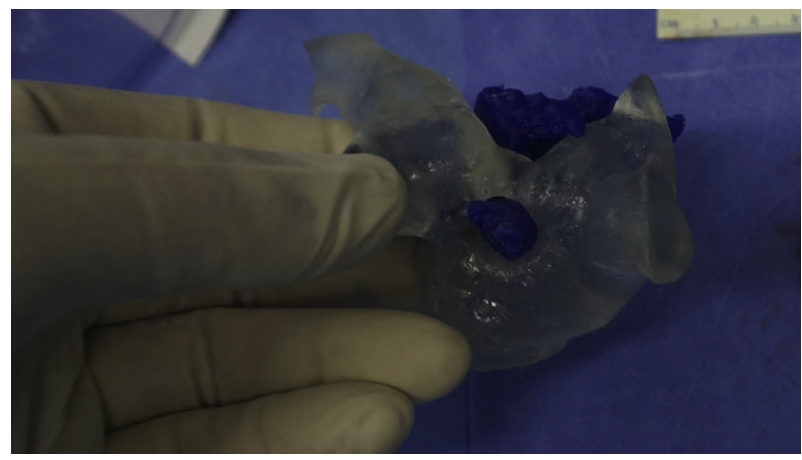

VIDEO 1. The case, from examination to discussion with the patient to the surgical procedure highlights, is shown in the video. Video available at: https://www.jtcvs.org/article/S0022-5223(18)32481-4/fulltext. adjacent to posterior mitral valve leaflet) were free of neoplastic tissue (Figure 2). Bovine pericardial patch was used to reconstruct the left atrium and the right superior

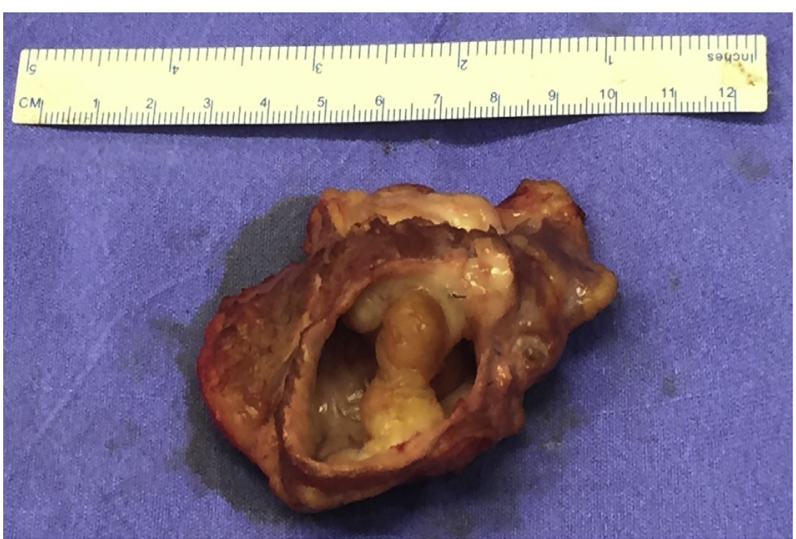

FIGURE 2. Tumor is seen invading the posterior superior left atrial endocardium, extending down close to the mitral valve annulus. Subtotal left atrial resection at the level of pulmonary veins was performed, preserving a 3- to 5-mm rim at the level of the mitral annulus. Free margins were seen on frozen-section analysis. 
pulmonary vein, with reimplantation of the other pulmonary veins and preservation of the mitral valve. No adjuncts were required, because the sutures were hemostatic. Histopathologic examination of the mass confirmed pathologic complete response with clear surgical margins. Crossclamp time was 150 minutes, and total cardiopulmonary bypass time was 165 minutes.

Postoperatively, the patient had pericarditis develop, which was satisfactorily treated with anti-inflammatory agents. There were no other significant complications, including no meaningful arrhythmias. He received postoperative anticoagulation, had maintenance with warfarin and aspirin for 1 year, and continued with a regimen of aspirin alone thereafter. He was discharged home on the ninth postoperative day. At 1 year after the tumor resection, he remains functionally well and free of local recurrence, but distant disease has been recently identified during surveillance imaging scans.

\section{DISCUSSION}

There are few reports on the utilization of 3D printing-a technology that manufactures the 3D object generated by complex radiologic imaging processing through layeron-layer addition of material to an existing surface-in planning for cardiac surgery. ${ }^{4}$ To our knowledge, this is the first report on utilization of a 3D printing model to perform an autotransplantation for cardiac sarcoma resection. Use of $3 \mathrm{D}$ printing might be advantageous for preoperative planning, teaching, surgical accuracy, and time saving in the operating room.

Costs and time needed for manufacturing the model may place important limitations on its use. ${ }^{5}$ Moreover,
3D printing may allow detailed understanding of the spatial relationship between the lesion and anatomic structures before surgery, which was critical in our case.

Although neoadjuvant therapy may help outcomes in cardiac sarcoma, ${ }^{6}$ complete tumor resection is the major prognostic factor for both symptom relief and survival in the treatment of primary cardiac disease and commonly demands complex reconstruction techniques. ${ }^{1,2}$ Autotransplantation-which consists of removing the heart, resecting the tumor with sufficient margins, reconstructing adjacent cardiac structures, and finally reimplanting the heart-is a safe technique being used increasingly by experienced surgeons, with a surgical mortality lower than $10 \%{ }^{3}$ In this case, the 3D model enabled better radiologic interpretation, discussion with the patient, and surgical planning, contributing to the successful procedure.

\section{References}

1. Ramlawi B, Leja MJ, Abu Saleh WK, Al Jabbari O, Benjamin R, Ravi V, et al Surgical treatment of primary cardiac sarcomas: review of a single-institution experience. Ann Thorac Surg. 2016;101:698-702.

2. Cusimano RJ. Surgical Management of Cardiac Tumors. Semin Diagn Pathol. 2008;25:76-81.

3. Ramlawi B, Al-Jabbari O, Blau LN, Davies MG, Bruckner BA, Blackmon SH, et al. Autotransplantation for the resection of complex left heart tumors. Ann Thorac Surg. 2014:98:863-8.

4. Valverde I. Three-dimensional printed cardiac models: applications in the field of medical education, cardiovascular surgery, and structural heart interventions. Rev Esp Cardiol (Engl Ed). 2017;70:282-91.

5. Martelli N, Serrano C, van den Brink H, Pineau J, Prognon P, Borget I, et al Advantages and disadvantages of 3-dimensional printing in surgery: a systematic review. Surgery. 2016;159:1485-500.

6. Abu Saleh WK, Ramlawi B, Shapira OM, Al Jabbari O, Ravi V, Benjamin R, et al. Improved outcomes with the evolution of a neoadjuvant chemotherapy approach to right heart sarcoma. Ann Thorac Surg. 2017;104:90-6. 